\title{
THE REPRESENTATION INVARIANTS OF 2-TERM SILTING COMPLEXES
}

\author{
YONGGANG HU
}

\begin{abstract}
Let $A$ be a finite dimensional algebra over a field $k$ and $\mathbf{P}$ be a 2 -term silting complex in $K^{b}(\operatorname{proj} A)$. In this paper, we investigate the representation dimension of $\operatorname{End}_{D^{b}(A)}(\mathbf{P})$ by using the silting theory. We show that if $\mathbf{P}$ is a separating silting complex with certain homological restriction, then rep.dim $A=$ rep.dim $\operatorname{End}_{D^{b}(A)}(\mathbf{P})$. This gives a proper generalization of the classical compare theorem of representation dimensions showed by Chen and $\mathrm{Hu}$. It is well-known that $\mathrm{H}^{0}(\mathbf{P})$ is a tilting $A / \operatorname{ann}_{A}(\mathbf{P})$-module. We also show that rep.dim $\operatorname{End}_{A}\left(\mathrm{H}^{0}(\mathbf{P})\right)=$ rep.dim $A / \operatorname{ann}_{A}(\mathbf{P})$ if $\mathbf{P}$ is a separating and splitting silting complex.
\end{abstract}

\section{INTRODUCTION}

The concept of representation dimension was first introduced by Auslander 1 in 1971. It is an important homological invariant in the representation theory. He proved that an artin algebra $A$ is representation-finite if and only if its representation dimension at most two. It means that the representation dimension gives a reasonable way of measuring how far an artin algebra is from being of finite representation type. In 1998, Reiten asked whether any artin algebra has a finite representation dimension. After this, Iyama 15 gave a positive answer to this question. Moreover, representation dimension of algebras is closely relative to other homological conjectures, such as the finitistic dimension conjecture. In particular, Igusa and Todorov [14] proved that if rep.dim $A \leq 3$, then $A$ has finite finitistic dimension. However, it was unsure that whether the representation dimension of an artin algebra can be greater than three, until in 2005 Rouquier 19 showed that representation dimension of an artin algebra may be arbitrarily large and constructed examples of algebras with representation dimension larger than or equal to four.

Up to now, the representation dimensions of several important classes of algebras are known to be at most three, such as hereditary algebras [1], torsionless-finite algebras [20, glued algebras 6, tilted algebras [2], quasi-tilted algebras [18, iterated tilted algebras [12, special biserial algebras 11], cluster-concealed algebras 5], and so on. In general, for a given artin algebra, it is difficult to know and compute the actual value of its representation dimension. However, there is a wise strategy to calculate the representation dimension by comparing two closely related algebras.

Date: February 12, 2020.

2000 Mathematics Subject Classification. Primary 16G10, 13D05; Secondary 13D30, 18E30.

Key words and phrases. Silting theory, Torsion pairs, Representation dimension, Tilting module.

This work was supported by CSC grant number 201906540017, from the China Scholarship Council. 
Then by the representation dimension of a known algebra, one can measure that of unknown algebra. Along this philosophy, it is nature to consider comparing the representation dimensions between algebra $A$ and $\operatorname{End}_{A}(T)$, where $T$ is a classical tilting right $A$-module. In this case, $T$ induces two torsion pairs $(\mathcal{T}(T), \mathcal{F}(T))$, $(\mathcal{X}(T), \mathcal{Y}(T))$ in $\bmod A$ and $\operatorname{modEnd}_{A}(T)$, respectively. These torsion pairs split the module categories into some different pieces. It turn out to be effective to compute the representation dimension by using torsion pairs, see 12 and 7 .

As a generalization of the classical tilting theory, the concept of silting complexes originated from Keller and Vossieck. In particular, Hoshino 13] showed that 2-term silting complexes can induce torsion pairs in module categories. More recently, Buan and Zhou 8] gave a generalization of the classical tilting theorem, called silting theorem. They described the relations of torison pairs between $\bmod A$ and $\bmod B$ by using the natural equivalences induced by Hom and Ext functors, where $B=\operatorname{End}_{D^{b}(A)}(\mathbf{P})$ and $\mathbf{P}$ is a 2 -term silting complex in $K^{b}(\operatorname{proj} A)$. It provides us with a basic framework to research the representation dimension by the silting theory. For more important homological results on the 2-term silting complexes, we refer the reader to 9 and 10$]$.

In this paper, we consider the representation invariants induced by 2 -term silting complexes. In details, we focus on when the representation dimensions of $A$ and $\operatorname{End}_{D^{b}(A)}(\mathbf{P})$ are coincide. Now, we present one of our main results as follows.

Theorem 1.1. Let $A$ be a finite dimensional algebra and let $\boldsymbol{P}$ be a 2-term separating silting complex in $K^{b}(\operatorname{proj} A)$ such that $\operatorname{Id}_{A} X \leq 1$ for each $X \in \mathcal{F}(\boldsymbol{P})$ and $B=\operatorname{End}_{D^{b}(A)}(\mathbf{P})$. Then rep.dim $B=$ rep.dim $A$.

Applying this result into the classical tilting theory, we can obtain 7 , Theorem 3.1]. Meanwhile, we give an example to illustrate that it is a proper generalization, see Example 4.3 .

On other hand, it is well-known that if $\mathbf{P}$ is a 2-term silting complex in $K^{b}(\operatorname{proj} A)$, then $\mathrm{H}^{0}(\mathbf{P})$ is a tilting $A / \operatorname{ann}_{A}(\mathbf{P})$-module, where $\operatorname{ann}_{A}(\mathbf{P})$ is the annihilator of $\mathrm{H}^{0}(\mathbf{P})$. In general, we know that $\operatorname{End}_{A}\left(\mathrm{H}^{0}(\mathbf{P})\right)$ is a factor algebra of $\operatorname{End}_{A}(\mathbf{P})$. It is interesting to consider to describe the relationship of the representation dimensions between $\operatorname{End}_{A}\left(\mathrm{H}^{0}(\mathbf{P})\right)$ and $A$. Thus, we have the following results.

Proposition 1.2. Let $A$ be a finite dimensional algebra and $\boldsymbol{P}$ be a 2-term splitting and separating silting complex in $K^{b}(\operatorname{proj} A)$. Then $\operatorname{rep} \cdot \operatorname{dimEnd}_{A}\left(\mathrm{H}^{0}(\mathbf{P})\right)=$ rep.dim $A / \operatorname{ann}_{A}(\mathbf{P})$.

The paper is organized as follows. In Section 2, we recall some well-known results on the silting theory and the representation dimension. In Section 3, we prove our main results. In Section 4, we provide some examples to illustrate that anyone of the conditions of Theorem 1.1 cannot be removed. In Section 5, we compare the representation dimensions of $\operatorname{End}_{A}\left(\mathrm{H}^{0}(\mathbf{P})\right)$ and $A / \operatorname{ann}_{A}(\mathbf{P})$.

\section{Preliminaries}

Let $A$ be a finite dimensional $k$-algebra with $k$ is a field. We denote by $\bmod A$ the category of finitely generated right $A$-modules. Let $D(-)=\operatorname{Hom}_{k}(-, k)$ be the $k$-duality. We denote by $\operatorname{proj} A$ the full subcategory of $\bmod A$ generated by the projective modules. Let $D^{b}(A)$ be the bounded derived category, with shift functor $\Sigma$ and $K^{b}(\operatorname{proj} A)$ the bounded homotopy category of finitely generated projective right $A$-modules. 
A complex $\mathbf{P}$ is said to be 2-term if $P^{i}=0$ for $i \neq 0,1$. Recall that a 2term complex $\mathbf{P}$ in $K^{b}(\operatorname{proj} A)$ is said to be silting if it satisfies the following two conditions

(1) $\operatorname{Hom}_{K^{b}(\operatorname{proj} A)}(\mathbf{P}, \Sigma \mathbf{P})=0$

(2) thick $\mathbf{P}=K^{b}(\operatorname{proj} A)$ where thick $\mathbf{P}$ is the smallest triangulated subcategory closed under direct summands containing $\mathbf{P}$.

In addition, if $\mathbf{P}$ satisfies $\operatorname{Hom}_{K^{b}(\operatorname{proj} A)}\left(\mathbf{P}, \Sigma^{-1} \mathbf{P}\right)=0$, then $\mathbf{P}$ is said to be tilting.

Let $\mathbf{P}$ be a 2 -term silting complex in $K^{b}(\operatorname{proj} A)$, and consider the following two full subcategories of $\bmod A$

$$
\begin{aligned}
& \mathcal{T}(\mathbf{P})=\left\{X \in \bmod A \mid \operatorname{Hom}_{D^{b}(A)}(\mathbf{P}, \Sigma X)=0\right\} \\
& \mathcal{F}(\mathbf{P})=\left\{Y \in \bmod A \mid \operatorname{Hom}_{D^{b}(A)}(\mathbf{P}, Y)=0\right\} .
\end{aligned}
$$

Theorem 2.1. 8 Let $\boldsymbol{P}$ be a 2-term silting complex in $K^{b}(\operatorname{proj} A)$, and let $B=$ $\operatorname{End}_{D^{b}(A)}(\mathbf{P})$.

(1) $\mathcal{C}(\boldsymbol{P})$ is an abelian category and the short exact sequences in $\mathcal{C}(\boldsymbol{P})$ are precisely the triangles in $D^{b}(A)$ all of whose vertices are objects in $\mathcal{C}(\boldsymbol{P})$.

(2) The pair $(\mathcal{T}(\mathbf{P}), \mathcal{F}(\mathbf{P}))$ is a torsion pair in $\bmod A$. The pair $(\Sigma \mathcal{F}(\mathbf{P}), \mathcal{T}(\mathbf{P}))$ is a torsion pair in $\mathcal{C}(\boldsymbol{P})$.

(3) $\operatorname{Hom}_{D^{b}(A)}(\mathbf{P},-): \mathcal{C}(\mathbf{P}) \rightarrow \bmod B$ is an equivalence of abelian categories.

(4) There is a triangle

$$
A \rightarrow \boldsymbol{P}^{\prime} \stackrel{f}{\rightarrow} \boldsymbol{P}^{\prime \prime} \rightarrow \Sigma A
$$

with $\boldsymbol{P}^{\prime}, \boldsymbol{P}^{\prime \prime}$ in add $\mathbf{P}$.

Consider the 2-term complex $\boldsymbol{Q}$ in $K^{b}(\operatorname{proj} B)$ induced by the map

$$
\operatorname{Hom}_{D^{b}(A)}(\mathbf{P}, f): \operatorname{Hom}_{D^{b}(A)}\left(\mathbf{P}, \mathbf{P}^{\prime}\right) \rightarrow \operatorname{Hom}_{D^{b}(A)}\left(\mathbf{P}, \mathbf{P}^{\prime \prime}\right) .
$$

(5) $\boldsymbol{Q}$ is a 2-term silting complex in $K^{b}(\operatorname{proj} B)$ such that

$$
\begin{aligned}
& \mathcal{T}(\mathbf{Q})=\mathcal{X}(\mathbf{P})=\operatorname{Hom}_{D^{b}(A)}(\mathbf{P}, \Sigma \mathcal{F}(\mathbf{P})) \\
& \mathcal{F}(\mathbf{Q})=\mathcal{Y}(\mathbf{P})=\operatorname{Hom}_{D^{b}(A)}(\mathbf{P}, \mathcal{T}(\mathbf{P})) .
\end{aligned}
$$

(6) There is an algebra epimorphism $\Phi_{\mathbf{P}}: A \rightarrow \bar{A}=\operatorname{End}_{D^{b}(B)}(\mathbf{Q})$.

(7) $\Phi_{\boldsymbol{P}}$ is an isomorphism if and only if $\boldsymbol{P}$ is tilting.

(8) Let $\Phi_{*}: \bmod \bar{A} \rightarrow \bmod A$ be the inclusion functor. Then one obtains the following picture, which also shows the quasi-inverse equivalences between the pair $(\mathcal{T}(\mathbf{P}), \mathcal{F}(\mathbf{P}))$ and $(\mathcal{T}(\mathbf{Q}), \mathcal{F}(\mathbf{Q}))$.

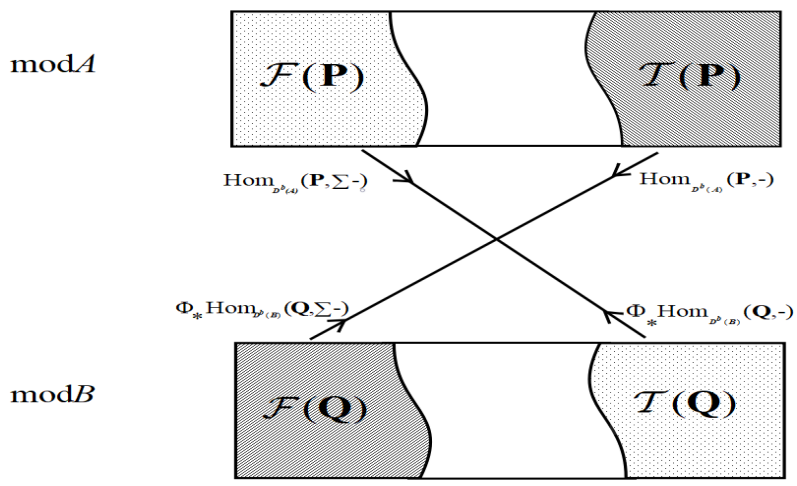


In what following, the symbol $\mathbf{Q}$ always denotes the induced complex $\mathbf{Q}$. It is a 2 -term silting complex in $K^{b}(\operatorname{proj} B)$ such that the induced pair $(\mathcal{T}(\mathbf{Q}), \mathcal{F}(\mathbf{Q}))=$ $(\mathcal{X}(\mathbf{P}), \mathcal{Y}(\mathbf{P}))$.

Definition 2.2. 8 Let $\mathbf{P}$ is a 2 -term silting complex in $K^{b}(\operatorname{proj} A)$.

(1) $\mathbf{P}$ is called splitting if the induced torsion pair $(\mathcal{X}(\mathbf{P}), \mathcal{Y}(\mathbf{P}))$ in $\bmod B$ is split.

(2) $\mathbf{P}$ is called separating if the induced torsion pair $(\mathcal{T}(\mathbf{P}), \mathcal{F}(\mathbf{P}))$ in $\bmod A$ is split.

Remark 2.3. 8 Let $\mathbf{P}$ be a 2-term silting complex in $K^{b}(\operatorname{proj} B)$.

(1) $\mathbf{P}$ is splitting if and only if $\operatorname{Ext}_{A}^{2}(\mathcal{T}(\mathbf{P}), \mathcal{F}(\mathbf{P}))=0$.

(2) If $\mathbf{P}$ is separating, then $\mathbf{P}$ is a tilting complex.

(3) Suppose that $\mathbf{P}$ is both splitting and separating. In this case, $\mathbf{Q}$ is a separating silting complex and so, it is a tilting complex. Then, $A \cong$ $\operatorname{End}_{D^{b}(B)}(\mathbf{Q})$. In this case, $(\mathcal{X}(\mathbf{Q}), \mathcal{Y}(\mathbf{Q}))=(\mathcal{T}(\mathbf{P}), \mathcal{F}(\mathbf{P}))$. Then $\mathbf{Q}$ is also a splitting silting complex.

Lemma 2.4. 8 Let $\boldsymbol{P}$ be a 2-term silting complex in $K^{b}(\operatorname{proj} A)$ and $(\mathcal{T}(\mathbf{P}), \mathcal{F}(\mathbf{P}))$ be the induced torsion pair in $\bmod A$. Then the following hold.

(1) For any $X \in \bmod A, X \in \operatorname{add} H^{0}(\mathbf{P})$ if and only if $X$ is Ext-projective in $\mathcal{T}(\boldsymbol{P})$.

(2) For any $X \in \bmod A, X \in \operatorname{add} H^{-1}(\nu \mathbf{P})$ if and only if $X$ is Ext-injective in $\mathcal{F}(\boldsymbol{P})$.

Let $M$ be a module in $\bmod A . M$ is said to be a generator of $\bmod A$ if $A \in \operatorname{add} M$. Dually, one can define the cogenerator. The representation of algebra $A$ is defined as

rep.dim $A=\inf \left\{\operatorname{gl} \cdot \operatorname{dim}\left(\operatorname{End}_{A}(M)\right) \mid M\right.$ a generator and cogenerator of $\left.\bmod A\right\}$.

Lemma 2.5. ([1], [11, Lemma 2.1]) Let $A$ be an algebra, $n$ be a non-negative integer at least 2 and $M$ be a generator-cogenerator for $\bmod A$. Then the following statements are equivalent:

(1) $\operatorname{gl} \cdot \operatorname{dim}\left(\operatorname{End}_{A}(M)\right) \leq n+2$,

(2) For each $A$-module $X$, there exists an exact sequence

$$
0 \rightarrow M_{n} \rightarrow M_{n-1} \rightarrow \cdots \rightarrow M_{0} \longrightarrow X \rightarrow 0
$$

with $M_{i}$ in $\operatorname{add} M$ for all $i$, such that the induced sequence

$0 \rightarrow \operatorname{Hom}_{A}\left(M, M_{n}\right) \rightarrow \cdots \rightarrow \operatorname{Hom}_{A}\left(M, M_{1}\right) \rightarrow \operatorname{Hom}_{A}(M, X) \rightarrow 0$

is exact.

(3) For each $A$-module $X$, there exists an exact sequence

$$
0 \rightarrow X \rightarrow M_{0} \rightarrow \cdots \rightarrow M_{1} \rightarrow M_{n} \rightarrow 0
$$

with $M_{i}$ in $\operatorname{add} M$ for all $i$, such that the induced sequence

$0 \rightarrow \operatorname{Hom}_{A}\left(M_{n}, M\right) \rightarrow \cdots \rightarrow \operatorname{Hom}_{A}\left(M_{0}, M\right) \rightarrow \operatorname{Hom}_{A}(X, M) \rightarrow 0$

An $A$-module $M$ is said to be an Aulsander generator of $\bmod A$ if it satisfies that gl.dim $\left(\operatorname{End}_{A}(M)\right)=$ rep.dim $A$. 


\section{MAin RESUlt}

In this section, we will compare the the representation dimensions of $A$ and $\operatorname{End}_{D^{b}(A)}(\mathbf{P})$.

The following result was proved in 13 , in the setting of abelian categories with arbitrary coproducts. Indeed, it is also true in our case. The proof of the following lemma has contained in [8]. For convenience, we provide the details of proof here.

Lemma 3.1. For any $X \in \bmod A, \operatorname{Hom}_{D^{b}(A)}\left(\mathbf{P}, \Sigma^{i} X\right)=0$ for any $i<0$ and $i>1$.

Proof. It is easy to check that $\operatorname{Hom}_{D^{b}(A)}\left(\mathbf{P}, \Sigma^{i} X\right)=0$ for any $i>1$. Now we prove the former case. Assume that $\mathbf{P}: P^{-1} \stackrel{d}{\rightarrow} P^{0}$ where all $P^{i}$ are finitely generated projective modules. Then there is a distinguished triangle

$$
P^{-1} \stackrel{-d}{\longrightarrow} P^{0} \rightarrow \mathbf{P} \rightarrow \Sigma P^{-1} .
$$

Applying the functor $\operatorname{Hom}_{D^{b}(A)}\left(-, \Sigma^{i} X\right)=0$ to the sequence 3.1. we have the following sequence

$\cdots \rightarrow \operatorname{Hom}_{D^{b}(A)}\left(\Sigma P^{-1}, \Sigma^{i} X\right) \rightarrow \operatorname{Hom}_{D^{b}(A)}\left(\mathbf{P}, \Sigma^{i} X\right) \rightarrow \operatorname{Hom}_{D^{b}(A)}\left(P^{0}, \Sigma^{i} X\right) \rightarrow \cdots$

Note that for any $i<0, \operatorname{Hom}_{D^{b}(A)}\left(\Sigma P^{-1}, \Sigma^{i} X\right)=\operatorname{Hom}_{D^{b}(A)}\left(P^{0}, \Sigma^{i} X\right)=0$. Therefore, $\operatorname{Hom}_{D^{b}(A)}\left(\mathbf{P}, \Sigma^{i} X\right)=0$ for any $i<0$.

Next, we shall character the right $B$-module $\operatorname{End}_{A}\left(\mathrm{H}^{0}(\mathbf{P})\right)$.

Lemma 3.2. Let $\boldsymbol{P}$ be a 2-term splitting silting complex in $K^{b}(\operatorname{proj} A)$ and $B=$ $\operatorname{End}_{D^{b}(A)}(\mathbf{P})$. Then the right $B$-module $\operatorname{End}_{A}\left(\mathrm{H}^{0}(\mathbf{P})\right)$ is projective. In particular, $B \cong \operatorname{Hom}_{D^{b}(A)}\left(\mathbf{P}, \mathrm{H}^{0}(\mathbf{P})\right) \oplus \operatorname{Hom}_{D^{b}(A)}\left(\mathbf{P}, \Sigma \mathrm{H}^{-1}(\mathbf{P})\right)$.

Proof. Let $X$ be an indecomposable right $B$-module. Since $\mathbf{P}$ is a splitting silting complex, $X \in \mathcal{X}(\mathbf{P})$ or $X \in \mathcal{Y}(\mathbf{P})$. Note that $\operatorname{End}_{A}\left(\mathrm{H}^{0}(\mathbf{P})\right) \cong \operatorname{Hom}_{D^{b}(A)}\left(\mathbf{P}, \mathrm{H}^{0}(\mathbf{P})\right)$ which is in $\mathcal{Y}(\mathbf{P})$ as right $B$-module since $\mathrm{H}^{0}(\mathbf{P}) \in \mathcal{T}(\mathbf{P})$. If $X \in \mathcal{X}(\mathbf{P})$, then, we get the isomorphism $\operatorname{Ext}_{B}^{1}\left(\operatorname{End}_{A}\left(\mathrm{H}^{0}(\mathbf{P})\right), X\right) \cong D \underline{\operatorname{Hom}}\left(\tau^{-1} X, \operatorname{End}_{A}\left(\mathrm{H}^{0}(\mathbf{P})\right)\right.$ by AR-formula. Since $(\mathcal{X}(\mathbf{P}), \mathcal{Y}(\mathbf{P}))$ is split and $X \in \mathcal{X}(\mathbf{P}), \tau^{-1} X \in \mathcal{X}(\mathbf{P})$ and hence, $\operatorname{Ext}_{B}^{1}\left(\operatorname{End}_{A}\left(\mathrm{H}^{0}(\mathbf{P}), X\right)=0\right.$. If $X \in \mathcal{Y}(\mathbf{P})$, then there exists a right $A$-module $X^{\prime} \in \mathcal{T}(\mathbf{P})$ such that $X \cong \operatorname{Hom}_{D^{b}(A)}\left(\mathbf{P}, X^{\prime}\right)$. For any short exact sequence

$$
0 \rightarrow \operatorname{Hom}_{D^{b}(A)}\left(\mathbf{P}, X^{\prime}\right) \rightarrow U \rightarrow \operatorname{Hom}_{D^{b}(A)}\left(\mathbf{P}, \mathrm{H}^{0}(\mathbf{P})\right) \rightarrow 0
$$

in $\operatorname{Ext}_{B}^{1}\left(\operatorname{End}_{A}\left(\mathrm{H}^{0}(\mathbf{P}), X\right), U \in \mathcal{Y}(\mathbf{P})\right.$ since the first and third terms are in $\mathcal{Y}(\mathbf{P})$ and there exists $U^{\prime} \in \mathcal{T}(\mathbf{P})$ such that $U \cong \operatorname{Hom}_{D^{b}(A)}\left(\mathbf{P}, U^{\prime}\right)$. It follows that there is an exact sequence

$$
0 \rightarrow X^{\prime} \rightarrow U^{\prime} \rightarrow \mathrm{H}^{0}(\mathbf{P}) \rightarrow 0
$$

in $\bmod A$. Note that $\mathrm{H}^{0}(\mathbf{P})$ is an Ext-projective module in $\mathcal{T}(\mathbf{P})$. Thus, the sequence 3.3 splits. It yields that the sequence 3.2 splits. Hence, we have that $\operatorname{Hom}_{D^{b}(A)}\left(\mathbf{P}, \mathrm{H}^{0}(\mathbf{P})\right)$ is projective in $\bmod B$.

Note that for any 2-term complex $\mathbf{Y}$ in $D^{b}(A)$, there is a triangle

$$
\Sigma \mathrm{H}^{-1}(\mathbf{Y}) \rightarrow \mathbf{Y} \rightarrow \mathrm{H}^{0}(\mathbf{Y}) \rightarrow \Sigma^{2} \mathrm{H}^{-1}(\mathbf{Y}) .
$$

Applying $\operatorname{Hom}_{D^{b}(A)}(\mathbf{P},-)$ to the triangle

$$
\Sigma \mathrm{H}^{-1}(\mathbf{P}) \rightarrow \mathbf{P} \rightarrow \mathrm{H}^{0}(\mathbf{P}) \rightarrow \Sigma^{2} \mathrm{H}^{-1}(\mathbf{P}),
$$


we get the following long exact sequence of right $B$-modules

$$
\begin{aligned}
\cdots & \rightarrow \operatorname{Hom}_{D^{b}(A)}\left(\mathbf{P}, \Sigma^{-1} \mathrm{H}^{0}(\mathbf{P})\right) \rightarrow \operatorname{Hom}_{D^{b}(A)}\left(\mathbf{P}, \Sigma \mathrm{H}^{-1}(\mathbf{P})\right) \rightarrow \operatorname{Hom}_{D^{b}(A)}(\mathbf{P}, \mathbf{P}) \\
& \rightarrow \operatorname{Hom}_{D^{b}(A)}\left(\mathbf{P}, \mathrm{H}^{0}(\mathbf{P})\right) \rightarrow \operatorname{Hom}_{D^{b}(A)}\left(\mathbf{P}, \Sigma^{2} \mathrm{H}^{-1}(\mathbf{P})\right) \rightarrow \cdots .
\end{aligned}
$$

By Lemma 3.1, we get a short exact sequence

$$
0 \rightarrow \operatorname{Hom}_{D^{b}(A)}\left(\mathbf{P}, \Sigma \mathrm{H}^{-1}(\mathbf{P})\right) \rightarrow \operatorname{Hom}_{D^{b}(A)}(\mathbf{P}, \mathbf{P}) \rightarrow \operatorname{Hom}_{D^{b}(A)}\left(\mathbf{P}, \mathrm{H}^{0}(\mathbf{P})\right) \rightarrow 0 .
$$

The result follows form that $\operatorname{Hom}_{D^{b}(A)}\left(\mathbf{P}, \mathrm{H}^{0}(\mathbf{P})\right)$ is projective.

Lemma 3.3. Let $\boldsymbol{P}$ be a 2-term tilting complex in $K^{b}(\operatorname{proj} A)$. Then $\nu \boldsymbol{P} \in \mathcal{C}(\boldsymbol{P})$. In this case, $\mathrm{H}^{0}(\nu \mathbf{P}) \in \mathcal{T}(\mathbf{P})$ and $\mathrm{H}^{-1}(\nu \mathbf{P}) \in \mathcal{F}(\mathbf{P})$.

Proof. It suffices to show that $\nu \mathbf{P} \in \mathrm{D}^{\leq 0}(\mathbf{P}) \cap \mathrm{D}^{\geq 0}(\mathbf{P})$. The result follows from the following equations

$$
\begin{aligned}
\operatorname{Hom}_{D^{b}(A)}\left(\mathbf{P}, \Sigma^{i} \nu \mathbf{P}\right) & =\mathrm{H}^{i} \operatorname{Hom}^{\bullet}(\mathbf{P}, \nu \mathbf{P}) \\
& \cong \mathrm{H}^{i} D\left(\mathbf{P}^{*} \otimes_{A}^{\bullet} \mathbf{P}\right) \\
& \cong \mathrm{H}^{i} D \operatorname{Hom}^{\bullet}(\mathbf{P}, \mathbf{P}) \\
& \cong D \mathrm{H}^{-i} \operatorname{Hom}^{\bullet}(\mathbf{P}, \mathbf{P}) \\
& \cong D \operatorname{Hom}_{D^{b}(A)}\left(\mathbf{P}, \Sigma^{-i} \mathbf{P}\right) .
\end{aligned}
$$

The rest results are from [4, Lemma 2.13].

Dually, we can describe the right $B$-module $\operatorname{Hom}_{D^{b}(A)}\left(\mathbf{P}, \Sigma \mathrm{H}^{-1}(\nu \mathbf{P})\right)$.

Lemma 3.4. Let $\boldsymbol{P}$ be a 2-term splitting silting complex in $K^{b}(\operatorname{proj} A)$ and $B=$ $\operatorname{End}_{D^{b}(A)}(\mathbf{P})$. Then the right $B$-module $\operatorname{Hom}_{D^{b}(A)}\left(\mathbf{P}, \Sigma \mathrm{H}^{-1}(\nu \mathbf{P})\right)$ is an injective module. In this case, $D B \cong \operatorname{Hom}_{D^{b}(A)}\left(\mathbf{P}, \Sigma \mathrm{H}^{-1}(\nu \mathbf{P})\right) \oplus \operatorname{Hom}_{D^{b}(A)}\left(\mathbf{P}, \mathrm{H}^{0}(\nu \mathbf{P})\right)$.

Proof. It is easy to check that $D B=\operatorname{Hom}_{D^{b}(A)}(\mathbf{P}, \nu \mathbf{P})$. Since $\mathrm{H}^{-1}(\nu \mathbf{P}) \in \mathcal{F}(\mathbf{P})$, we know that $\operatorname{Hom}_{D^{b}(A)}\left(\mathbf{P}, \Sigma H^{-1}(\nu \mathbf{P})\right) \in \mathcal{X}(\mathbf{P})$. If $Y \in \mathcal{X}(\mathbf{P})$, then, we get the isomorphism

$$
\operatorname{Ext}_{B}^{1}\left(Y, \operatorname{Hom}_{D^{b}(A)}\left(\mathbf{P}, \Sigma \mathrm{H}^{-1}(\nu \mathbf{P})\right)\right) \cong D \overline{\operatorname{Hom}}_{B}\left(\operatorname{Hom}_{D^{b}(A)}\left(\mathbf{P}, \Sigma \mathrm{H}^{-1}(\nu \mathbf{P})\right), \tau Y\right)
$$

by AR-formula. Since $(\mathcal{X}(\mathbf{P}), \mathcal{Y}(\mathbf{P}))$ is split and $Y \in \mathcal{Y}(\mathbf{P}), \tau Y \in \mathcal{Y}(\mathbf{P})$ and hence, $\operatorname{Ext}_{B}^{1}\left(\mathcal{Y}, \operatorname{Hom}_{D^{b}(A)}\left(\mathbf{P}, \Sigma \mathrm{H}^{-1}(\nu \mathbf{P})\right)\right)=0$. If $Y \in \mathcal{X}(\mathbf{P})$, then there exists a right $A$-module $Y^{\prime} \in \mathcal{F}(\mathbf{P})$ such that $X \cong \operatorname{Hom}_{D^{b}(A)}\left(\mathbf{P}, \Sigma Y^{\prime}\right)$. For any short exact sequence

$$
0 \rightarrow \operatorname{Hom}_{D^{b}(A)}\left(\mathbf{P}, \Sigma \mathrm{H}^{-1}(\nu \mathbf{P})\right) \rightarrow V \rightarrow \operatorname{Hom}_{D^{b}(A)}\left(\mathbf{P}, \Sigma Y^{\prime}\right) \rightarrow 0
$$

in $\operatorname{Ext}_{B}^{1}\left(Y, \operatorname{Hom}_{D^{b}(A)}\left(\mathbf{P}, \Sigma \mathrm{H}^{-1}(\nu \mathbf{P})\right)\right), V \in \mathcal{X}(\mathbf{P})$ since the first and third terms are in $\mathcal{X}(\mathbf{P})$ and there exists $V^{\prime} \in \mathcal{F}(\mathbf{P})$ such that $V \cong \operatorname{Hom}_{D^{b}(A)}\left(\mathbf{P}, \Sigma V^{\prime}\right)$. It follows that there is an exact sequence

$$
0 \rightarrow \mathrm{H}^{-1}(\nu \mathbf{P}) \rightarrow V^{\prime} \rightarrow Y^{\prime} \rightarrow 0
$$

in $\mathcal{F}(\mathbf{P})$. Note that $\mathrm{H}^{-1}(\nu \mathbf{P})$ is an Ext-injective module in $\mathcal{F}(\mathbf{P})$. Thus, the sequence (3.5) splits. It yields that the sequence (3.4) splits. Thus, we know that $\operatorname{Hom}_{D^{b}(A)}\left(\mathbf{P}, \Sigma \mathrm{H}^{-1}(\nu \mathbf{P})\right)$ is injective in $\bmod B$.

Applying $\operatorname{Hom}_{D^{b}(A)}(\mathbf{P},-)$ to the triangle

$$
\Sigma \mathrm{H}^{-1}(\nu \mathbf{P}) \rightarrow \nu \mathbf{P} \rightarrow \mathrm{H}^{0}(\nu \mathbf{P}) \rightarrow \Sigma^{2} \mathrm{H}^{-1}(\nu \mathbf{P}),
$$


we get the following long exact sequence of right $B$-modules

$$
\begin{aligned}
\cdots & \rightarrow \operatorname{Hom}_{D^{b}(A)}\left(\mathbf{P}, \Sigma^{-1} \mathrm{H}^{0}(\nu \mathbf{P})\right) \rightarrow \operatorname{Hom}_{D^{b}(A)}\left(\mathbf{P}, \Sigma \mathrm{H}^{-1}(\nu \mathbf{P})\right) \rightarrow \operatorname{Hom}_{D^{b}(A)}(\mathbf{P}, \nu \mathbf{P}) \\
& \rightarrow \operatorname{Hom}_{D^{b}(A)}\left(\mathbf{P}, \mathrm{H}^{0}(\nu \mathbf{P})\right) \rightarrow \operatorname{Hom}_{D^{b}(A)}\left(\mathbf{P}, \Sigma^{2} \mathrm{H}^{-1}(\nu \mathbf{P})\right) \rightarrow \cdots
\end{aligned}
$$

By Lemma 3.1, we get a short exact sequence

$0 \rightarrow \operatorname{Hom}_{D^{b}(A)}\left(\mathbf{P}, \Sigma \mathrm{H}^{-1}(\nu \mathbf{P})\right) \rightarrow \operatorname{Hom}_{D^{b}(A)}(\mathbf{P}, \nu \mathbf{P}) \rightarrow \operatorname{Hom}_{D^{b}(A)}\left(\mathbf{P}, \mathrm{H}^{0}(\nu \mathbf{P})\right) \rightarrow 0$.

The result follows form that $\operatorname{Hom}_{D^{b}(A)}\left(\mathbf{P}, \mathrm{H}^{-1}(\nu \mathbf{P})\right)$ is injective.

Proposition 3.5. Let $A$ be a finite dimensional algebra and let $\boldsymbol{P}$ be a 2-term silting complex in $K^{b}(\operatorname{proj} A)$ such that $\operatorname{Id}_{A} X \leq 1$ for each $X \in \mathcal{F}(\boldsymbol{P})$ and $B=$ End $_{D^{b}(A)}(\mathbf{P})$. Then $\boldsymbol{P}$ is separating if and only if for any $M \in \mathcal{X}(\boldsymbol{P}), \operatorname{Pd}_{B} M \leq 1$.

Proof. For the necessity, it is enough to prove that $\operatorname{Pd}_{B} M \leq 1$, for any module $M \in \mathcal{T}(\mathbf{Q})$.

By the assumption, we know that $\mathbf{P}$ is a splitting silting complex. Since $\mathbf{P}$ is a separating silting complex, then $\mathbf{P}$ is a tilting complex and hence, $\mathbf{Q}$ is a tilting complex. Then $A \cong \operatorname{End}_{D^{b}(B)}(\mathbf{Q})$. Moreover, the torsion pairs $(\mathcal{T}(\mathbf{P}), \mathcal{F}(\mathbf{P}))$ and $(\mathcal{T}(\mathbf{Q}), \mathcal{F}(\mathbf{Q}))$ are split in $\bmod A$ and $\bmod B$, respectively.

Since $(\mathcal{T}(\mathbf{Q}), \mathcal{F}(\mathbf{Q}))$ is split, for any indecomposable $N \in \bmod B, N \in \mathcal{T}(\mathbf{Q})$ or $N \in \mathcal{F}(\mathbf{Q})$. If $N \in \mathcal{F}(\mathbf{Q})$, then

$$
\begin{aligned}
\operatorname{Ext}_{B}^{2}(M, N) & \cong \operatorname{Hom}_{D^{b}(B)}\left(M, \Sigma^{2} N\right) \\
& =\operatorname{Hom}_{D^{b}(B)}(M, \Sigma(\Sigma N))(\text { by Theorem 2.1 }(1)) \\
& \cong \operatorname{Ext}_{A}^{1}\left(\operatorname{Hom}_{D^{b}(B)}(\mathbf{Q}, M), \operatorname{Hom}_{D^{b}(B)}(\mathbf{Q}, \Sigma N)\right)(\text { by Theorem 2.1 }(2))
\end{aligned}
$$

Note that $\operatorname{Hom}_{D^{b}(B)}(\mathbf{Q}, \Sigma N) \in \mathcal{T}(\mathbf{P})$ and $\operatorname{Hom}_{D^{b}(B)}(\mathbf{Q}, M) \in \mathcal{F}(\mathbf{P})$. Since $(\mathcal{T}(\mathbf{P}), \mathcal{F}(\mathbf{P}))$ is split, $\operatorname{Ext}_{A}^{1}\left(\operatorname{Hom}_{D^{b}(B)}(\mathbf{Q}, M), \operatorname{Hom}_{D^{b}(B)}(\mathbf{Q}, \Sigma N)\right)=0$. Thus, $\operatorname{Ext}_{B}^{2}(M, N)=0$. If $N \in \mathcal{T}(\mathbf{Q})$, then

$$
\begin{aligned}
\operatorname{Ext}_{B}^{2}(M, N) & \cong \operatorname{Hom}_{D^{b}(B)}\left(M, \Sigma^{2} N\right) \\
& \cong \operatorname{Hom}_{D^{b}(A)}\left(\operatorname{Hom}_{D^{b}(B)}(\mathbf{Q}, M), \Sigma^{2} \operatorname{Hom}_{D^{b}(B)}(\mathbf{Q}, N)\right) \\
& \cong \operatorname{Ext}_{A}^{2}\left(\operatorname{Hom}_{D^{b}(B)}(\mathbf{Q}, M), \operatorname{Hom}_{D^{b}(B)}(\mathbf{Q}, N)\right) .
\end{aligned}
$$

Since $\operatorname{Hom}_{D^{b}(B)}(\mathbf{Q}, N) \in \mathcal{F}(\mathbf{P})$, by the assumption on $\mathcal{F}(\mathbf{P})$, we have that

$$
\operatorname{Ext}_{A}^{2}\left(\operatorname{Hom}_{D^{b}(B)}(\mathbf{Q}, M), \operatorname{Hom}_{D^{b}(B)}(\mathbf{Q}, N)\right)=0
$$

and hence $\operatorname{Ext}_{B}^{2}(M, N)=0$. Then the claim holds.

For the sufficiency, assume that $X \in \mathcal{F}(\mathbf{P})$ and $Y \in \mathcal{T}(\mathbf{P})$. It suffices to show that $\operatorname{Ext}_{A}^{1}(X, Y)=0$. Indeed, we have the following isomorphisms

$$
\begin{aligned}
\operatorname{Ext}_{A}^{1}(X, Y) & \cong \operatorname{Hom}_{D^{b}(A)}(X, \Sigma Y) \\
& \cong \operatorname{Hom}_{D^{b}(A)}\left(\Sigma X, \Sigma^{2} Y\right) \\
& \cong \operatorname{Hom}_{D^{b}(B)}\left(\operatorname{Hom}_{D^{b}(A)}(\mathbf{P}, \Sigma X), \Sigma^{2} \operatorname{Hom}_{D^{b}(A)}(\mathbf{P}, Y)\right) \\
& \cong \operatorname{Ext}_{B}^{2}\left(\operatorname{Hom}_{D^{b}(A)}(\mathbf{P}, \Sigma X), \operatorname{Hom}_{D^{b}(A)}(\mathbf{P}, Y)\right) .
\end{aligned}
$$

Note that $\operatorname{Hom}_{D^{b}(A)}(\mathbf{P}, \Sigma X) \in \mathcal{X}(\mathbf{P})$. By the assumption, we know that the projective dimension of $\operatorname{Hom}_{D^{b}(A)}(\mathbf{P}, \Sigma X)$ at most 1. It implies that $\operatorname{Ext}_{A}^{1}(X, Y)=0$.

Dually, one can prove the following result. 
Proposition 3.6. Let $A$ be a finite dimensional algebra and let $\boldsymbol{P}$ be a 2-term silting complex in $K^{b}(\operatorname{proj} A)$ such that $\operatorname{Pd}_{A} X \leq 1$ for each $X \in \mathcal{T}(\boldsymbol{P})$ and $B=$ $\operatorname{End}_{D^{b}(A)}(\mathbf{P})$. Then $\boldsymbol{P}$ is separating if and only if for any $N \in \mathcal{Y}(\boldsymbol{P}), \operatorname{id}_{B} N \leq 1$.

In what follows, for convenience, we denote by $\mathcal{H}(-)$ the functor $\operatorname{Hom}_{D^{b}(A)}(\mathbf{P},-)$ and by $\mathcal{E}(-)$ the functor $\operatorname{Hom}_{D^{b}(A)}(\mathbf{P}, \Sigma-)$.

Lemma 3.7. Let $A$ be a finite dimensional algebra and let $\boldsymbol{P}$ be a 2-term separating silting complex in $K^{b}(\operatorname{proj} A)$ such that $\operatorname{Id}_{A} X \leq 1$ for each $X \in \mathcal{F}(\boldsymbol{P})$ and $B=$ $\operatorname{End}_{D^{b}(A)}(\mathbf{P})$. Suppose that $M$ is a generator of $\bmod A$ and $N=B \oplus \mathcal{H}(M) \oplus Y$ with $Y \in \mathcal{X}(\boldsymbol{P})$. Then $\operatorname{Pd}_{\operatorname{End}_{B}(N)} \operatorname{Hom}_{B}(N, \mathcal{H}(U)) \leq \operatorname{Pd}_{\operatorname{End}_{A}(M)} \operatorname{Hom}_{B}(M, U)$ for any $U \in \mathcal{T}(\boldsymbol{P})$.

Proof. If $\operatorname{Pd}_{\operatorname{End}_{A}(M)} \operatorname{Hom}_{B}(M, U)=\infty$, then the result holds. Otherwise, we assume that $\operatorname{Pd}_{\operatorname{End}_{A}(M)} \operatorname{Hom}_{B}(M, U)=n<\infty$. Since $M$ is a generator of $\bmod A$, there is an $\operatorname{add} M$-resolution of $U$, that is, there is a long exact sequence

$$
0 \rightarrow M_{n} \stackrel{f_{n}}{\longrightarrow} \cdots \rightarrow M_{1} \stackrel{f_{1}}{\longrightarrow} M_{0} \stackrel{f_{0}}{\longrightarrow} U \rightarrow 0
$$

such that it keeps exact after applying $\operatorname{Hom}_{A}\left(M^{\prime},-\right)$ with $M^{\prime} \in \operatorname{add} M$. Then there are a family of short each short exact sequences

$$
0 \rightarrow K_{i+1} \stackrel{\eta_{i}}{\longrightarrow} M_{i} \stackrel{\overline{f_{i}}}{\longrightarrow} K_{i} \rightarrow 0
$$

for $i=0,1, \cdots, n-1$, where $K_{0}=U, K_{n}=M_{n}, K_{j}=\operatorname{Ker} f_{j}$ for $j=1,2, \cdots, n-1$ and $\overline{f_{i}}$ are right add $M$-approximations. Applying the functor $\mathcal{H}$ to these sequences, we obtain a long exact sequence

$$
0 \rightarrow \mathcal{H}\left(K_{i+1}\right) \stackrel{\mathcal{H}\left(\eta_{i}\right)}{\longrightarrow} \mathcal{H}\left(M_{i}\right) \stackrel{\mathcal{H}\left(\overline{f_{i}}\right)}{\longrightarrow} \mathcal{H}\left(K_{i}\right) \rightarrow \mathcal{E}\left(K_{i+1}\right) \rightarrow \mathcal{E}\left(M_{i}\right) \rightarrow \mathcal{E}\left(K_{i}\right) \rightarrow 0
$$

Set $\Omega_{i}=\operatorname{Coker} \mathcal{H}\left(\overline{f_{i}}\right)$. Then we get a long exact sequence

$$
0 \rightarrow \Omega_{i} \rightarrow \mathcal{E}\left(K_{i+1}\right) \rightarrow \mathcal{E}\left(M_{i}\right) \rightarrow \mathcal{E}\left(K_{i}\right) \rightarrow 0
$$

Note that $\mathcal{E}\left(K_{i+1}\right), \mathcal{E}\left(M_{i}\right), \mathcal{E}\left(K_{i}\right)$ are in $\mathcal{X}(\mathbf{P})$. Then by Proposition 3.5 , we know that the projective dimensions of $\mathcal{E}\left(K_{i+1}\right), \mathcal{E}\left(M_{i}\right), \mathcal{E}\left(K_{i}\right)$ are at most 1 . Thus, $\operatorname{Pd}_{B} \Omega_{i} \leq 1$. Assume that $0 \rightarrow B_{i}^{1} \stackrel{\beta_{i}}{\longrightarrow} B_{i}^{0} \stackrel{\alpha_{i}}{\longrightarrow} \Omega_{i} \rightarrow 0$ is the projective resolution of $\Omega_{i}$ with $B_{i}^{j} \in \operatorname{add} B$. Then we obtain the following commutative diagram

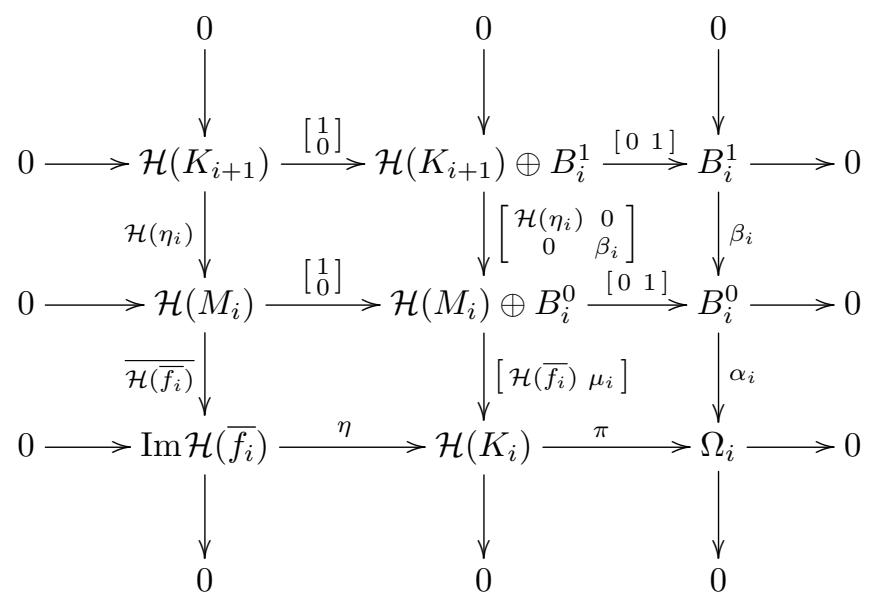


where the existence of the morphism $\mu_{i}$ is from the projectiveness of $B_{i}^{0}$. Let $\sigma_{i}=\left[\mathcal{H}\left(\overline{f_{i}}\right) \mu_{i}\right]$. Then we have a short exact sequence

$$
0 \rightarrow \mathcal{H}\left(K_{i+1}\right) \oplus B_{i}^{1} \rightarrow \mathcal{H}\left(M_{i}\right) \oplus B_{i}^{0} \stackrel{\sigma_{i}}{\rightarrow} \mathcal{H}\left(K_{i}\right) \rightarrow 0 .
$$

Next, we claim that the above sequence keeps exact after applying $\operatorname{Hom}_{B}\left(N^{\prime},-\right)$ for any $N^{\prime} \in \operatorname{add} N$. It suffices to show that the induced map

$$
\operatorname{Hom}_{B}\left(N^{\prime}, \mathcal{H}\left(M_{i}\right) \oplus B_{i}^{0}\right) \rightarrow \operatorname{Hom}_{B}\left(N^{\prime}, \mathcal{H}\left(K_{i}\right)\right)
$$

is surjective for $N^{\prime} \in \operatorname{add} Y$ or $N^{\prime} \in \operatorname{add} \mathcal{H}(M)$. If $N^{\prime} \in \operatorname{add} Y$, then we know that $\operatorname{Hom}_{B}\left(N^{\prime}, \mathcal{H}\left(K_{i}\right)\right)=0$ since $N^{\prime} \in \mathcal{X}(\mathbf{P})$ and $\mathcal{H}\left(K_{i}\right) \in \mathcal{Y}(\mathbf{P})$. Now assume $N^{\prime} \in$ $\operatorname{add} \mathcal{H}(M)$ and $N^{\prime}$ is a nonzero object. Then there is a module $M^{\prime} \in \operatorname{add} M \cap \mathcal{T}(\mathbf{P})$ such that $N^{\prime}=\mathcal{H}\left(M^{\prime}\right)$. Then we have the following isomorphisms

$$
\begin{aligned}
\operatorname{Hom}_{B}\left(N^{\prime}, \mathcal{H}\left(K_{i}\right)\right) & =\operatorname{Hom}_{B}\left(\mathcal{H}\left(M^{\prime}\right), \mathcal{H}\left(K_{i}\right)\right) \\
& \cong \operatorname{Hom}_{B}\left(\mathcal{H}\left(M^{\prime}\right), \mathcal{H}\left(t K_{i}\right)\right) \\
& \cong \operatorname{Hom}_{B}\left(M^{\prime}, t K_{i}\right) \\
& \cong \operatorname{Hom}_{B}\left(M^{\prime}, K_{i}\right)
\end{aligned}
$$

Thus, for any $g \in \operatorname{Hom}_{B}\left(N^{\prime}, \mathcal{H}\left(K_{i}\right)\right)$, there is a morphism $g^{\prime}: M^{\prime} \rightarrow K_{i}$ such that $g=\mathcal{H}\left(g^{\prime}\right)$. Since $\overline{f_{i}}$ is a right add $M$-approximation, there is a morphism $h: M^{\prime} \rightarrow M_{i}$ such that $\overline{f_{i}} h=g^{\prime}$. Then we have the following commutative diagram

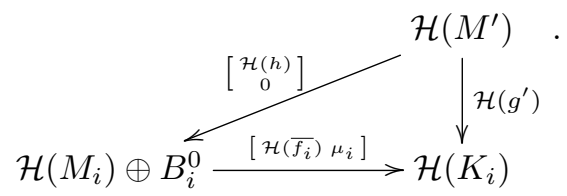

Then the claim holds.

Now, we can construct a long exact sequence

$$
0 \rightarrow N_{n} \rightarrow \cdots \rightarrow N_{1} \rightarrow N_{0} \rightarrow \mathcal{H}(U) \rightarrow 0
$$

such that it keeps exact after applying $\operatorname{Hom}_{B}(N,-)$, where $N_{n}=\mathcal{H}\left(M_{n}\right) \oplus B_{n-1}^{1}$, $N_{0}=\mathcal{H}\left(M_{0}\right) \oplus B_{0}^{0}$ and $N_{i}=\mathcal{H}\left(M_{i}\right) \oplus B_{i}^{0} \oplus B_{i-1}^{1}$ for $i=1,2, \cdots, n-1$. Therefore, $\operatorname{Pd}_{\operatorname{End}_{B}(N)} \operatorname{Hom}_{B}(N, \mathcal{H}(U)) \leq n$.

Dually, we have the following lemma.

Lemma 3.8. Let $A$ be a finite dimensional algebra and let $\boldsymbol{P}$ be a 2-term separating silting complex in $K^{b}(\operatorname{proj} A)$ such that $\operatorname{Pd}_{A} X \leq 1$ for each $X \in \mathcal{T}(\boldsymbol{P})$ and $B=\operatorname{End}_{D^{b}(A)}(\mathbf{P})$. Suppose that $M$ is a cogenerator of $\bmod A$ and $N=$ $D B \oplus \mathcal{E}(M) \oplus Y$ with $Y \in \mathcal{F}(\boldsymbol{P})$. Let $\Lambda=\operatorname{End}_{B}(N)^{o p}$ and $\Gamma=\operatorname{End}_{A}(M)^{o p}$. Then $\operatorname{Pd}_{\Lambda} \operatorname{Hom}_{B}(\mathcal{E}(U), N) \leq \operatorname{Pd}_{\Gamma} \operatorname{Hom}_{B}(U, M)$ for any $U \in \mathcal{F}(\boldsymbol{P})$.

Lemma 3.9. Let $A$ be a finite dimensional algebra and $\boldsymbol{P}$ be a 2-term separating silting complex in $K^{b}(\operatorname{proj} A)$ and $B=\operatorname{End}_{D^{b}(A)}(\mathbf{P})$. If $Y$ is a non-projective indecomposable direct summand of $\mathrm{H}^{0}(\mathbf{P})$ and $0 \rightarrow \tau Y \rightarrow U \rightarrow Y \rightarrow 0$ is an almost split sequence, then $U \in \operatorname{add}\left(\mathrm{H}^{0}(\mathbf{P}) \oplus \mathrm{H}^{-1}(\nu \mathbf{P})\right)$.

Proof. Since $\mathbf{P}$ is separating, we can assume that $U \cong K \oplus L$ with $K \in \mathcal{T}(\mathbf{P})$ and $L \in \mathcal{F}(\mathbf{P})$. The the prove can be divided into two cases.

Case 1. Let $K^{\prime}$ be an indecomposable direct summand of $K$. To prove $K^{\prime} \in$ $\operatorname{addH}^{0}(\mathbf{P})$, it suffices to show that $K^{\prime}$ is Ext-projective in $\mathcal{T}(\mathbf{P})$ by Lemma 2.4. If 
$K^{\prime}$ is projective, then there is nothing to prove. Assume that $K^{\prime}$ is not projective. Note that there is an irreducible map $\tau Y \rightarrow K^{\prime}$. Then there is an irreducible map $\tau K^{\prime} \rightarrow \tau Y$. Since $\tau \mathrm{H}^{0}(\mathbf{P}) \in \mathcal{F}(\mathbf{P})$ and $\mathcal{F}(\mathbf{P})$ is closed under the predecessors, $\tau K^{\prime} \in \mathcal{F}(\mathbf{P})$. Hence, $K^{\prime}$ is Ext-projective in $\mathcal{T}(\mathbf{P})$.

Case 2. Let $L^{\prime}$ be an indecomposable direct summand of $L$. To prove $L^{\prime} \in$ $\left.\operatorname{addH}^{-1}(\nu \mathbf{P})\right)$, it suffices to show that $K^{\prime}$ is Ext-injective in $\mathcal{F}(\mathbf{P})$ by Lemma 2.4 If $L^{\prime}$ is injective, then there is nothing to prove. Assume that $L^{\prime}$ is not injective. Note that there is an irreducible map $L^{\prime} \rightarrow Y$. Then there is an irreducible map $Y \rightarrow \tau^{-1} L^{\prime}$. Since $\mathrm{H}^{0}(\mathbf{P}) \in \mathcal{T}(\mathbf{P})$ and $\mathcal{T}(\mathbf{P})$ is closed under the successors, $\tau^{-1} L^{\prime} \in$ $\mathcal{T}(\mathbf{P})$. Hence, $L^{\prime}$ is Ext-injective in $\mathcal{F}(\mathbf{P})$.

Lemma 3.10. Let $A$ be a finite dimensional algebra and $\boldsymbol{P}$ be a 2-term silting complex in $K^{b}(\operatorname{proj} A)$ such that $\operatorname{Id}_{A} X \leq 1$ for each $X \in \mathcal{F}(\boldsymbol{P})$ and $B=$ $\operatorname{End}_{D^{b}(A)}(\mathbf{P})$. Then $\operatorname{Hom}_{B}(\mathcal{E}(I), \mathcal{E}(X))=0$ where $I \in \mathcal{F}(\boldsymbol{P})$ is an injective module, $X \notin \operatorname{addH}^{-1}(\nu \mathbf{P})$ and $X \in \mathcal{F}(\mathbf{P})$ is indecomposable.

Proof. It suffices to show that $\operatorname{Hom}_{A}(I, X)=0$. Assume that $\operatorname{Hom}_{A}(I, X) \neq 0$. Then for any nonzero morphism $u: I \rightarrow X$. It is easy to see that $u$ is not injective. If $u$ is surjective, then there is a short exact sequence $0 \rightarrow \operatorname{Ker} u \rightarrow I \rightarrow X \rightarrow 0$ in $\mathcal{F}(\mathbf{P})$. By the assumption on $\mathcal{F}(\mathbf{P})$, we know that $X$ is injective and hence Ext-injective in $\mathcal{F}(\mathbf{P})$. It is impossible. Hence, $u$ is not surjective. We consider the short exact $0 \rightarrow \operatorname{Ker} u \rightarrow I \rightarrow \operatorname{Im} u \rightarrow 0$. Then $\operatorname{Im} u$ is an injective module. Since $\operatorname{Im} u$ is a nonzero injective module, the inclusion map $\operatorname{Im} u \rightarrow X$ is split. It makes a contradiction. Therefore $\operatorname{Hom}_{A}(I, X)=0$.

Lemma 3.11. Let $A$ be a finite dimensional algebra and $\boldsymbol{P}$ be a 2-term separating silting complex in $K^{b}(\operatorname{proj} A)$ such that $\operatorname{Id}_{A} X \leq 1$ for each $X \in \mathcal{F}(\boldsymbol{P})$ and $B=$ $\operatorname{End}_{D^{b}(A)}(\mathbf{P})$. Then $\operatorname{Hom}_{B}\left(\mathcal{E}\left(\tau \mathrm{H}^{0}(\mathbf{P})\right), \mathcal{E}(X)\right)=0$ where $X \notin \operatorname{addH}^{-1}(\nu \mathbf{P})$ and $X \in \mathcal{F}(\mathbf{P})$.

Proof. Assume that $X$ is indecomposable. It has shown that $\mathcal{E}\left(\mathrm{H}^{-1}(\nu \mathbf{P})\right)$ is an injective $B$-module by Lemma 3.4. Now assume that $Z$ is is a non-projective indecomposable direct summand of $\mathrm{H}^{0}(\mathbf{P})$. Then there is an AR-sequence

$$
0 \longrightarrow \tau Z \stackrel{\left[\begin{array}{l}
f \\
g
\end{array}\right]}{\longrightarrow} T \oplus Q \longrightarrow Z \longrightarrow 0 .
$$

with $T \in \operatorname{addH}^{0}(\mathbf{P})$ and $Q \in \operatorname{addH}^{-1}(\nu \mathbf{P})$.

Next, we claim that $\mathcal{E}(g): \mathcal{E}(\tau Z) \rightarrow \mathcal{E}(Q)$ is a minimal left almost split sequence. It is easy to check that $\mathcal{E}(g)$ is a left minimal morphism. Next we shall show that $\mathcal{E}(g)$ is a left almost split morphism. Let $W$ be an indecomposable $B$-module and $h: \mathcal{E}(\tau Z) \rightarrow W$ be not split monomorphism. Clearly, $W \in \mathcal{X}(\mathbf{P})$ and hence $W \cong$ $\mathcal{E}(V)$ for some indecomposable $A$-module $V \in \mathcal{F}(\mathbf{P})$. Thus, there is a morphism $h^{\prime}: \tau Z \rightarrow V$ such that $\mathcal{E}\left(h^{\prime}\right)=h$. Since $\left[\begin{array}{l}f \\ g\end{array}\right]$ is a left almost split morphism, we have the following commutative diagram

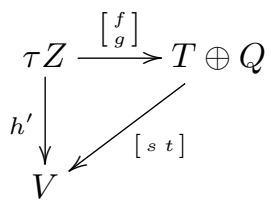


Note that $\operatorname{Hom}_{A}(T, V)=0$ since $T \in \mathcal{T}(\mathbf{P})$. Thus, $s=0$ and so, $h^{\prime}=t g$. Then $h=\mathcal{E}\left(h^{\prime}\right)=\mathcal{E}(t) \mathcal{E}(g)$.

Now, we focus on $Q$. Note that $\mathrm{H}^{-1}(\nu \mathbf{P})=\tau \mathrm{H}^{0}(\mathbf{P}) \oplus P$ where $P \in \mathcal{F}(\mathbf{P})$ is a projective $A$-module. If $P \in \operatorname{add} \tau \mathrm{H}^{0}(\mathbf{P})$, then $Q=\tau Z^{\prime}$ for some $Z^{\prime} \in \operatorname{add} \tau \mathrm{H}^{0}(\mathbf{P})$. Suppose that $P \notin \operatorname{add} \tau \mathrm{H}^{0}(\mathbf{P})$. In this case, we claim that $P$ is also an injective $A$-module. Since $\tau^{-1} \mathrm{H}^{-1}(\nu \mathbf{P}) \in \operatorname{addH}^{0}(\mathbf{P})$. Then $\tau^{-1} P \in \mathrm{H}^{0}(\mathbf{P})$. If $\tau^{-1} P$ is a nonzero module, then $\tau \tau^{-1} P \cong P \in \operatorname{add} \tau \mathrm{H}^{0}(\mathbf{P})$. It yields a contradiction. Thus, the claim holds. In this case, $Q \cong \tau Z^{\prime \prime} \oplus I$ where $Z^{\prime \prime} \in \operatorname{addH}^{0}(\mathbf{P})$ and $I \in \operatorname{add} P$.

We assume that $Q \cong \tau Z^{\prime \prime} \oplus I$. Now, we can prove that $\operatorname{Hom}_{B}(\mathcal{E}(\tau Z), \mathcal{E}(X))=0$ for $X \notin \operatorname{addH}^{-1}(\nu \mathbf{P})$ and $X \in \mathcal{F}(\mathbf{P})$. Let $u \in \operatorname{Hom}_{B}(\mathcal{E}(\tau Z), \mathcal{E}(X))$. Then $u$ is not spit monomorphism. Hence, we have the following commutative diagram

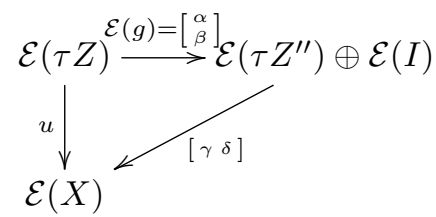

By Lemma 3.10, $\delta=0$ and so, $\gamma \alpha=u$. Since $\mathcal{E}(\tau Z) \in \operatorname{add} \mathcal{E}\left(\mathrm{H}^{-1}(\nu \mathbf{P})\right), \mathcal{E}(\tau Z)$ is injective. It follows that $\mathcal{E}(Q) \cong \mathcal{E}(\tau Z) / \operatorname{Soc} \mathcal{E}(g)$. Then the length $l(\mathcal{E}(Q))$ of $\mathcal{E}(Q)$ smaller than that of $\mathcal{E}(\tau Z)$. Using the induction on the length of $l(\tau Z)$, we know that $\operatorname{Hom}_{B}(\mathcal{E}(\tau Z), \mathcal{E}(X))=0$. It completes the proof.

Dually, we have the following result.

Lemma 3.12. Let $A$ be a finite dimensional algebra and $\boldsymbol{P}$ be a 2-term separating silting complex in $K^{b}(\operatorname{proj} A)$ such that $\operatorname{Pd}_{A} X \leq 1$ for each $X \in \mathcal{T}(\boldsymbol{P})$ and $B=$ $\operatorname{End}_{D^{b}(A)}(\mathbf{P})$. Then $\operatorname{Hom}_{B}\left(\mathcal{H}(X), \mathcal{H}\left(\mathrm{H}^{0}(\mathbf{P})\right)\right)=0$ where $X \notin \operatorname{addH}^{0}(\mathbf{P})$ and $X \in$ $\mathcal{T}(\mathbf{P})$.

Now, we are in position to prove our main result.

Theorem 3.13. Let $A$ be a finite dimensional algebra and let $\boldsymbol{P}$ be a 2-term separating silting complex in $K^{b}(\operatorname{proj} A)$ such that $\operatorname{Id}_{A} X \leq 1$ for each $X \in \mathcal{F}(\boldsymbol{P})$ and $B=\operatorname{End}_{D^{b}(A)}(\mathbf{P})$. Then rep.dim $B=$ rep.dim $A$.

Proof. Assume that $\mathbf{P}=P_{-1} \rightarrow P_{0}$. Consider the following exact sequence

$$
0 \rightarrow \mathrm{H}^{-1}(\nu \mathbf{P}) \rightarrow \nu P_{-1} \rightarrow \nu P_{0} \rightarrow \mathrm{H}^{0}(\nu \mathbf{P}) \rightarrow 0 .
$$

By the assumption on $\mathcal{F}(\mathbf{P})$ and $\mathrm{H}^{-1}(\nu \mathbf{P}) \in \mathcal{F}(\mathbf{P})$, then $H^{0}(\nu \mathbf{P})$ is an injective $A$ module. Now, let $M$ be the Auslander generator of $A$ such that gl.dimEnd ${ }_{A}(M)=$ rep.dim $A=n+2$.

Since $\mathbf{P}$ is a separating silting complex, we have $M \cong M_{\mathcal{T}} \oplus M_{\mathcal{F}}$ with $M_{\mathcal{T}} \in$ $\operatorname{add} M \cap \mathcal{T}(\mathbf{P})$ and $M_{\mathcal{F}} \in \operatorname{add} M \bigcap \mathcal{F}(\mathbf{P})$. Then by Lemma 3.3, $H^{0}(\nu \mathbf{P}) \in \operatorname{add} M_{\mathcal{T}}$. Now, we set a $B$-module

$$
N=B \oplus \mathcal{H}\left(M_{\mathcal{T}}\right) \oplus \mathcal{E}\left(M_{\mathcal{F}}\right) \oplus \mathcal{E}\left(H^{-1}(\nu \mathbf{P})\right) .
$$

By Lemma 3.4 , we know that $D B=\mathcal{E}\left(\mathrm{H}^{-1}(\nu \mathbf{P})\right) \oplus \mathcal{H}\left(\mathrm{H}^{0}(\nu \mathbf{P})\right)$. Thus, $D B \in \operatorname{add} N$. It implies that $N$ is a generator and cogenerator of $\bmod B$. Let $\Lambda=\operatorname{End}_{B}(N)$ and $\Gamma=\operatorname{End}_{A}(M)$. Next, we shall prove that gl.dim $\Lambda \leq$ gl.dim $\Gamma$. It suffices to show that $\operatorname{Pd}_{\Lambda} \operatorname{Hom}_{B}(N, X) \leq n$ for any indecomposable $B$-module $X$. Since $(\mathcal{X}(\mathbf{P}), \mathcal{Y}(\mathbf{P}))$ is a splitting torsion pair, $X \in \mathcal{X}(\mathbf{P})$ or $X \in \mathcal{Y}(\mathbf{P})$. If $X \in \mathcal{Y}(\mathbf{P})$, 
then there exists a module $U \in \mathcal{T}(\mathbf{P})$ such that $X \cong \mathcal{H}(U)$. By Lemma 3.7, we have $\operatorname{Pd}_{\Lambda} \operatorname{Hom}_{B}(N, \mathcal{H}(U)) \leq \operatorname{Pd}_{\Gamma} \operatorname{Hom}_{A}(M, U) \leq n$. If $X \in \mathcal{X}(\mathbf{P})$, then there exists a module $V \in \mathcal{F}(\mathbf{P})$ such that $X \cong \mathcal{E}(V)$. If $V \in \operatorname{addH}^{-1}(\nu \mathbf{P})$, then $\operatorname{Hom}_{B}(N, \mathcal{E}(V)) \in \operatorname{add} \Lambda$ and so, $\operatorname{Pd}_{\Lambda} \operatorname{Hom}_{B}(N, X)=0 \leq n$. Assume that $V \notin$ $\operatorname{addH}^{-1}(\nu \mathbf{P})$. We assume that $m \leq n$ is the maximal integer such that there is a minimal add $M$-resolution of $V$

$$
0 \rightarrow M_{m} \rightarrow \cdots \rightarrow M_{1} \rightarrow M_{0} \rightarrow V \rightarrow 0
$$

with all $M_{i} \in \operatorname{add} M$. In other words, for each $0 \leq i \leq m-1$, we have a short exact sequence

$$
0 \rightarrow K_{i+1} \rightarrow M_{i} \stackrel{f_{i}}{\longrightarrow} K_{i} \rightarrow 0
$$

where $f_{i}$ is a right minimal add $M$-approximation, $K_{0}=V$ and $K_{m}=M_{m}$. Since $\mathcal{F}(\mathbf{P})$ is closed under the predecessors, $K_{i}$ and $M_{i}$ are in $\mathcal{F}(\mathbf{P})$. Hence, any $K_{i}$ does not lie in $\operatorname{addH}^{-1}(\nu \mathbf{P})$ since $\mathrm{H}^{-1}(\nu \mathbf{P})$ is Ext-injective in $\mathcal{F}(\mathbf{P})$. Applying $\mathcal{H}$ to these short exact sequences, we have

$$
0 \rightarrow \mathcal{E}\left(K_{i+1}\right) \rightarrow \mathcal{E}\left(M_{i}\right) \stackrel{\mathcal{E}\left(f_{i}\right)}{\longrightarrow} \mathcal{E}\left(K_{i}\right) \rightarrow 0
$$

for $0 \leq i \leq m-1$.

Next, we claim that these above induced sequences keep exact after applying $\operatorname{Hom}_{B}\left(N^{\prime},-\right)$ for each indecomposable direct summand $N^{\prime}$ of $N$. Since $\mathbf{P}$ is splitting, $N^{\prime} \in \mathcal{Y}(\mathbf{P})$ or $N^{\prime} \in \mathcal{X}(\mathbf{P})$. If $N^{\prime} \in \mathcal{Y}(\mathbf{P})$, then by AR-formula, we have $\operatorname{Ext}_{B}^{1}\left(N^{\prime}, \mathcal{E}\left(K_{i+1}\right)\right)=D \overline{\operatorname{Hom}_{B}}\left(\mathcal{E}\left(K_{i+1}\right), \tau N^{\prime}\right)=0$ for $0 \leq i \leq m-1$ since $\tau N^{\prime} \in \mathcal{Y}(\mathbf{P})$ and $\mathcal{E}\left(K_{i+1}\right) \in \mathcal{X}(\mathbf{P})$. Thus, in this case, the claim holds. Now, we assume that $N^{\prime} \in \mathcal{X}(\mathbf{P})$. If $N^{\prime} \in \mathcal{E}\left(M_{\mathcal{F}}\right)$, then there exists $N^{\prime \prime} \in \operatorname{add} M_{\mathcal{F}}$ such that $\mathcal{E}\left(N^{\prime \prime}\right) \cong N^{\prime}$. Then we have the following commutative diagram

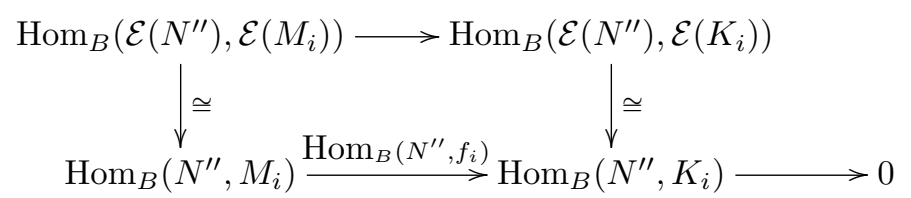

Thus, the upper row is surjective and the claim holds. We consider $N^{\prime} \in \mathcal{E}\left(\mathrm{H}^{-1}(\nu \mathbf{P})\right)$. Note that $\mathrm{H}^{-1}(\nu \mathbf{P}) \cong \tau \mathrm{H}^{0}(\mathbf{P}) \oplus P$ with $P \in \operatorname{add} A \bigcap \mathcal{F}(\mathbf{P}) \subseteq \operatorname{add} M_{\mathcal{F}}$. If $N^{\prime} \in$ $\operatorname{add} \mathcal{E}(P)$, then there is noting to prove. We assume that $N^{\prime} \in \operatorname{add} \mathcal{E}\left(\tau \mathrm{H}^{0}(\mathbf{P})\right)$. By Lemma 3.11, we know that $\operatorname{Hom}_{B}\left(N^{\prime}, \mathcal{E}\left(K_{i}\right)\right)=0$. Hence, the claim holds.

Therefore, there is a long exact sequence

$$
0 \rightarrow \operatorname{Hom}_{B}\left(N, \mathcal{E}\left(M_{m}\right)\right) \rightarrow \cdots \rightarrow \operatorname{Hom}_{B}\left(N, \mathcal{E}\left(M_{0}\right)\right) \rightarrow \operatorname{Hom}_{B}(N, \mathcal{E}(V)) \rightarrow 0
$$

where all $\mathcal{E}\left(M_{i}\right) \in \operatorname{add} N$. This implies that $\operatorname{Pd}_{\Lambda} \operatorname{Hom}_{B}(N, \mathcal{E}(V)) \leq m \leq n$ and so $\operatorname{Pd}_{\Lambda} \operatorname{Hom}_{B}(N, X) \leq n$ for any $X \in \bmod B$. Then, gl.dimEnd ${ }_{B}(N) \leq$ $n+2=$ gl.dimEnd $A(M)=$ rep.dim $A$. Therefore, $\operatorname{rep} \cdot \operatorname{dim} B \leq$ rep.dim $A$.

Assume that $\Theta$ is an Aulsander generator of $\bmod B$ and the induced silting complex $\mathbf{Q}=Q_{-1} \stackrel{\sigma}{\rightarrow} Q_{0}$ with $Q_{i} \in \operatorname{proj} B$. Since $\mathbf{Q}$ is a separating silting complex, we can write $\Theta=\Theta_{\mathcal{T}} \oplus \Theta_{\mathcal{F}}$ where $\Theta_{\mathcal{T}} \in \operatorname{add} \Theta \bigcap \mathcal{T}(\mathbf{Q})$ and $\Theta_{\mathcal{F}} \in \operatorname{add} \Theta \bigcap \mathcal{F}(\mathbf{Q})$. We consider the following exact sequence in $\bmod B$

$$
0 \rightarrow \mathrm{H}^{-1}(\mathbf{Q}) \rightarrow Q_{-1} \stackrel{\sigma}{\rightarrow} Q_{0} \rightarrow \mathrm{H}^{0}(\mathbf{Q}) \rightarrow 0 .
$$


Since $\mathrm{H}^{0}(\mathbf{Q}) \in \mathcal{T}(\mathbf{Q})=\mathcal{X}(\mathbf{P})$, by Proposition 3.5, we have $\operatorname{Pd}_{B} \mathrm{H}^{0}(\mathbf{Q}) \leq 1$ and so, $\mathrm{H}^{-1}(\mathbf{Q})$ is a projective $B$-module. By 13 , Proposition 5.4], $\mathrm{H}^{-1}(\mathbf{Q}) \in \operatorname{add} \Theta_{\mathcal{F}}$. Now, we define an $A$-module

$$
\Theta^{\prime}=D B \oplus \mathcal{E}\left(\Theta_{\mathcal{F}}\right) \oplus \mathcal{H}\left(\Theta_{\mathcal{T}}\right) \oplus \mathcal{H}\left(\mathrm{H}^{0}(\mathbf{Q})\right) .
$$

By Lemma 3.2 we know that $A \cong \operatorname{End}_{B}(\mathbf{Q})=\mathcal{H}\left(\mathrm{H}^{0}(\mathbf{Q})\right) \oplus \mathcal{E}\left(\mathrm{H}^{-1}(\mathbf{Q})\right) \in \operatorname{add}^{\prime}$. Hence, $\Theta^{\prime}$ is a generator and cogenerator of $\bmod A$. Dually, we apply Lemma 2.5 (3), Proposition 3.6. Lemma 3.8 and Lemma 3.12 to the 2-term silting complex $\mathbf{Q}$ in $K^{b}(\operatorname{proj} B)$ and $\Theta^{\prime}$. Then we obtain rep.dim $A=$ rep.dim $\operatorname{End}_{B}(\mathbf{Q}) \leq \operatorname{rep} \cdot \operatorname{dim} B$. Therefore, rep.dim $A=$ rep.dim $B$.

The following result is directly from Theorem 3.13 .

Corollary 3.14. Let $A$ be a finite dimensional hereditary algebra. If $\boldsymbol{P}$ is a 2-term separating stilting complex with $B=\operatorname{End}_{D^{b}(A)}(\mathbf{P})$, then rep.dim $B \leq 3$. Moreover, if $A$ is representation-finite, then $B$ is also representation-finite.

Let $T$ be a classical tilting right $A$-module. If one take $\mathbf{P}$ as the projective resolution of $T$, then $\mathbf{P}$ is a 2-term silting complex such that $\mathcal{T}(\mathbf{P})=\mathcal{T}(T)=$ $\operatorname{KerExt}_{A}^{1}(T,-)$ and $\mathcal{F}(\mathbf{P})=\mathcal{F}(T)=\operatorname{KerHom}_{A}(T,-)$. Moreover, one can see that $(\mathcal{X}(\mathbf{P}), \mathcal{Y}(\mathbf{P}))=(\mathcal{X}(\mathcal{T}), \mathcal{Y}(\mathcal{T}))$ in $\bmod B$, where $B=\operatorname{End}_{A}(T)=\operatorname{End}_{D^{b}(A)}(\mathbf{P})$. It is easy to prove that $\mathbf{P}$ is separating when $T$ is separating. If $T$ is splitting, then $\operatorname{Id}_{A} X \leq 1$ for any $X \in \mathcal{F}(\mathcal{T})=\mathcal{F}(\mathbf{P})$. Thus, we have the following consequence.

Corollary 3.15. 7, Theorem 3.1] Let $A$ be a finite dimensional algebra and $B=$ $\operatorname{End}_{A}(T)$. If $T$ is a separating and splitting tilting $A$-module, then rep.dim $A=$ rep.dim $B$.

\section{EXAMPLES}

Firstly, we would like to give two examples to illustrate that anyone of the conditions of Theorem 3.13 cannot be removed.

Example 4.1. Let $A$ be a path algebra over a filed $k$ and its quiver $Q_{A}$ given by

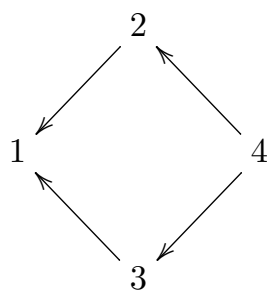

Set a right $A$-module $T=1 \oplus \begin{array}{rrr}4 & 4 \\ 3 & \oplus \begin{array}{l}2 \\ 1\end{array} & 1\end{array} \oplus 4$. It is easy to see that $T$ is a tilting module. Let $\mathbf{P}$ be the projective resolution of $T$. Then $\mathbf{P}$ is a 2-term silting complex. Since $A$ is a hereditary algebra, $\mathbf{P}$ is a splitting silting complex. Then 
$B=\operatorname{End}_{D^{b}(A)}(\mathbf{P})=\operatorname{End}_{A}(T)$ is given by the quiver $Q_{B}$

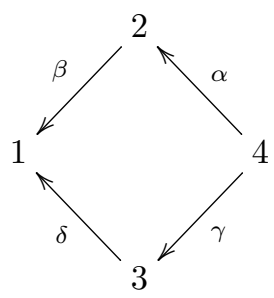

with relations $\alpha \beta=0$ and $\gamma \delta=0$. The AR-quiver of $\bmod B$ is given by

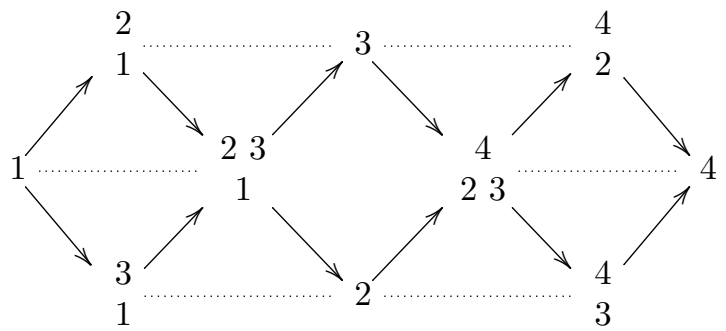

where the dot lines are the $\tau$-orbits. The induced splitting torsion pair $(\mathcal{X}(\mathbf{P})$, $\mathcal{Y}(\mathbf{P}))$ in $\bmod B$ is given by

$$
\begin{aligned}
& \mathcal{X}(\mathbf{P})=\left\{\begin{array}{ll}
4 & 4 \\
2 & 3
\end{array}, 4\right\} \\
& \mathcal{Y}(\mathbf{P})=\left\{\begin{array}{ccccccc}
1, & 2 & 1
\end{array}, \begin{array}{c}
3 \\
1
\end{array}, \begin{array}{c}
23 \\
1
\end{array}, 3, \quad 2, \quad \begin{array}{c}
43 \\
3
\end{array}\right\}
\end{aligned}
$$

Note that $\operatorname{pd}_{B} S(4)=2>1$ where $S(4) \in \mathcal{X}(\mathbf{P})$ is a simple $B$-module corresponding to the vertex 4 of $Q_{B}$. Thus, by Lemma 3.5, $\mathbf{P}$ is not a separating silting complex. It is easy to see that rep.dim $B=2<$ rep.dim $A=3$.

The following example given by 4. Due to this example, we can see that the homological dimension restriction on $\mathcal{F}(\mathbf{P})$ cannot be removed.

Example 4.2. Let $A$ be a $k$-algebra given by the following quiver

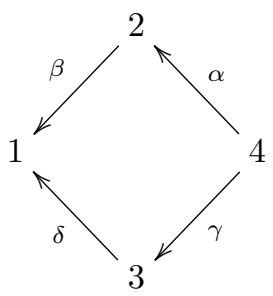

with relations $\alpha \beta=0$ and $\gamma \delta=0$. Then the AR-quiver of $\bmod A$ is form as the graph 4.1. There is a splitting torsion pair $(\mathcal{T}, \mathcal{F})$ in $\bmod A$

$$
\begin{aligned}
& \mathcal{T}=\left\{\begin{array}{ccc}
4 & 4 & 4 \\
23 & 2 & 3
\end{array}, 4\right\} \\
& \mathcal{F}=\left\{\begin{array}{lll}
1, & 2 \\
1 & 3 & 1
\end{array}, \begin{array}{c}
23 \\
1
\end{array}, 3,2\right\} .
\end{aligned}
$$


The 2-term silting complex $\mathbf{P}$ is given by the direct sums of the following complexes in $K^{b}(\operatorname{proj} A)$

$$
\begin{aligned}
& \mathbf{P}_{1}=0 \rightarrow \begin{array}{c}
4 \\
23
\end{array} \\
& \mathbf{P}_{2}=\begin{array}{l}
3 \\
1
\end{array} \rightarrow \begin{array}{c}
4 \\
23
\end{array} \\
& \mathbf{P}_{3}=\begin{array}{c}
2 \\
1
\end{array} \rightarrow \begin{array}{c}
4 \\
23
\end{array} \\
& \mathbf{P}_{4}=1 \rightarrow 0 \text {. }
\end{aligned}
$$

In this case, $\mathcal{T}=\mathcal{T}(\mathbf{P})$ and $\mathcal{F}=\mathcal{F}(\mathbf{P})$. Thus, the silting complex $\mathbf{P}$ is separating. Moreover, the endomorphism algebra $B=\operatorname{End}_{D^{b}(A)}(\mathbf{P})$ is a path algebra given by the following quiver, whose underlying graph is a Euclidean graph of $\widetilde{A_{3}}$

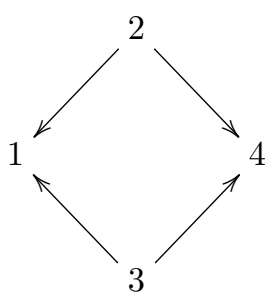

Thus, $\operatorname{End}_{D^{b}(A)}(\mathbf{P})$ is representation-infinite. Thus, rep.dim $A=2<$ rep.dim $B=3$. Note that $\operatorname{id}_{A} S(1)=2$ where $S(1) \in \mathcal{F}(\mathbf{P})$ is a simple $A$-module corresponding to the vertex 1 . Therefore, the separating silting complex $\mathbf{P}$ does not satisfy the homological dimension restriction on $\mathcal{F}(\mathbf{P})$.

Secondly, we should illustrate that Theorem 3.13 gives a proper generalization of the result in [7. It means that one can find a 2-term silting complex $\mathbf{P}$ satisfying the assumption in Theorem 3.13 , but it cannot be induced by a tilting module. In fact, this silting complex is not rare.

Example 4.3. Let $A$ be a path algebra given by the following quiver

$$
3 \longrightarrow 2 \longrightarrow 1
$$

The AR-quiver is given by

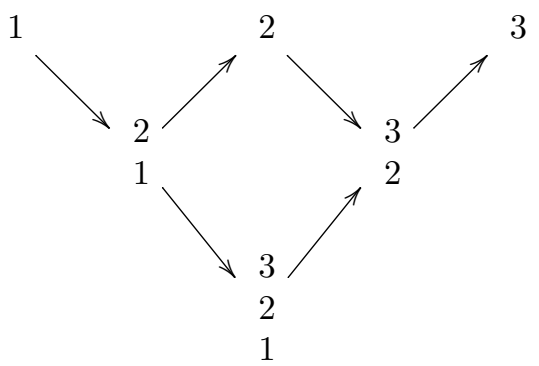


Then one can take a splitting torsion pair $(\mathcal{T}, \mathcal{F})$ as follows.

$$
\begin{aligned}
& \mathcal{T}=\left\{\begin{array}{lll}
2, & 3 \\
& 2 & 3
\end{array}\right\} \\
& \mathcal{F}=\left\{\begin{array}{lll}
1, & 2 \\
1 & , & 2 \\
1
\end{array}\right\}
\end{aligned}
$$

Note that this torsion pair cannot be induced by tilting modules in $\bmod A$. Let $\mathbf{P}$ be a 2-term complex given by the direct sums of the following complex in $K^{b}(\operatorname{proj} A)$

$$
\mathbf{P}_{1}=1 \rightarrow \begin{array}{r}
2 \\
1
\end{array} \quad \mathbf{P}_{2}=\begin{array}{r}
1 \\
\end{array} \quad \begin{array}{r}
3 \\
2 \\
1
\end{array} \quad \mathbf{P}_{3}=\begin{aligned}
& 3 \\
& 2 \\
& 1
\end{aligned} \rightarrow 0
$$

It is easy to check that $\mathbf{P}$ is a silting complex such that $\mathcal{T}=\mathcal{T}(\mathbf{P})$ and $\mathcal{F}=\mathcal{F}(\mathbf{P})$. Thus, $\mathbf{P}$ is a separating silting complex. It is not difficult to see that $\mathbf{P}$ is not a projective resolution of a tilting module. Since $A$ is a hereditary algebra, by Theorem $3.13 \operatorname{End}_{D^{b}(A)}(\mathbf{P})$ is also representation-finite.

\section{The Representation dimension of $\operatorname{End}_{A}\left(\mathrm{H}^{0}(\mathbf{P})\right)$}

Let $A$ be a finite dimensional algebra and $\mathbf{P}$ be a silting complex in $K^{b}(\operatorname{proj} A)$. We set $\mathfrak{A}=\operatorname{ann}_{A}(\mathbf{P})$ the annihilator of $\mathrm{H}^{0}(\mathbf{P})$. It is well-known that $\mathrm{H}^{0}(\mathbf{P})$ is a tilting $A / \mathfrak{A}$-module see 4 , Lemma 3.1]. We denote by Fac $M$ is a full subcategory consisting of all factor modules of the finite copies of a $A$-module $M$, i.e

$$
\operatorname{Fac} M=\left\{X \in \bmod \Lambda \mid M^{(n)} \rightarrow X \text { for some integer } n\right\} .
$$

Proposition 5.1. Let $A$ be a finite dimensional algebra and $\boldsymbol{P}$ be a 2-term splitting and separating silting complex in $K^{b}(\operatorname{proj} A)$. Then $\mathrm{H}^{0}(\mathbf{P})$ is a splitting and separating tilting $A / \mathfrak{A}$-module.

Proof. Firstly, we prove that $\mathrm{H}^{0}(\mathbf{P})$ is a splitting tilting $A / \mathfrak{A}$-module.

The canonical full embedding $\bmod A / \mathfrak{A} \hookrightarrow \bmod A$ induces $\operatorname{Fac}\left(\mathrm{H}^{0}(\mathbf{P})_{A / \mathfrak{A}}\right)=$ $\operatorname{Fac}\left(\mathrm{H}^{0}(\mathbf{P})_{A}\right)$. Since $\mathrm{H}^{0}(\mathbf{P})$ is a tilting $A / \mathfrak{A}$-module, $\mathcal{T}\left(\mathrm{H}^{0}(\mathbf{P})_{A / \mathfrak{A}}\right)=\operatorname{Fac}\left(\mathrm{H}^{0}(\mathbf{P})_{A / \mathfrak{A}}\right)$. It is well-known that $\mathcal{T}(\mathbf{P})=\operatorname{Fac}\left(\mathrm{H}^{0}(\mathbf{P})_{A}\right)$. Hence, $\mathcal{T}\left(\mathrm{H}^{0}(\mathbf{P})_{A / \mathfrak{A}}\right)=\mathcal{T}(\mathbf{P})$. For any indecomposable $Y \in \bmod A / \mathfrak{A}$. There is a short exact sequence in $\bmod A / \mathfrak{A}$

$$
0 \rightarrow Y \rightarrow \mathrm{E}(Y) \rightarrow \Omega_{A / \mathfrak{A}}^{-1}(Y) \rightarrow 0
$$

where $\mathrm{E}(Y)$ is the injective hull of $Y$ and $\Omega_{A / \mathfrak{A}}^{-1}(Y)$ is the 1-th cosyzygy of $Y$. Note that $D(A / \mathfrak{A}) \in \mathcal{T}\left(\mathrm{H}^{0}(\mathbf{P})_{A / \mathfrak{A}}\right)$. Then, $\Omega_{A / \mathfrak{A}}^{-1}(Y) \in \mathcal{T}\left(\mathrm{H}^{0}(\mathbf{P})_{A / \mathfrak{A}}\right)$ and so $\Omega_{A / \mathfrak{A}}^{-1}(Y) \in$ $\mathcal{T}(\mathbf{P})$ as a right $A$-module.

Assume that $Y \in \mathcal{F}\left(\mathrm{H}^{0}(\mathbf{P})_{A / \mathfrak{A}}\right)$. We claim that $\operatorname{id}_{A / \mathfrak{A}} Y \leq 1$. It suffices to prove that $\operatorname{Ext}_{A / \mathfrak{A}}^{2}(X, Y)=0$ for any indecomposable $A / \mathfrak{A}$-module $X$.

Since $\mathbf{P}$ is separating, $(\mathcal{T}(\mathbf{P}), \mathcal{F}(\mathbf{P}))$ is splitting. Note that $X$ is also an indecomposable $A$-module. Then $X$ is either in $\mathcal{F}(\mathbf{P})$ or $\mathcal{T}(\mathbf{P})$.

Assume that $X \in \mathcal{F}(\mathbf{P})$. Applying $\operatorname{Hom}_{A / \mathfrak{A}}(X,-)$ to the sequence 5.1], we have the exact sequence

$$
\operatorname{Ext}_{A / \mathfrak{A}}^{1}\left(X, \Omega_{A / \mathfrak{A}}^{-1}(Y)\right) \rightarrow \operatorname{Ext}_{A / \mathfrak{A}}^{2}(X, Y) \rightarrow \operatorname{Ext}_{A / \mathfrak{A}}^{2}(X, \mathrm{E}(Y))
$$


Note that there is a natural monoic map $\operatorname{Ext}_{A / \mathfrak{A}}^{1}\left(X, \Omega_{A / \mathfrak{A}}^{-1}(Y)\right) \hookrightarrow \operatorname{Ext}_{A}^{1}\left(X, \Omega_{A / \mathfrak{A}}^{-1}(Y)\right)$ see 17. Since $\Omega_{A / \mathfrak{A}}^{-1}(Y) \in \mathcal{T}(\mathbf{P})$, we have $\operatorname{Ext}_{A}^{1}\left(X, \Omega_{A / \mathfrak{A}}^{-1}(Y)\right)=0$ and hence, $\operatorname{Ext}_{A / \mathfrak{A}}^{1}\left(X, \Omega_{A / \mathfrak{A}}^{-1}(Y)\right)=0$. It implies that $\operatorname{Ext}_{A / \mathfrak{A}}^{2}(X, Y)=0$.

Now we assume $X \in \mathcal{T}(\mathbf{P})$. By [4, Lemma 2.13(2)], we have

$$
\begin{aligned}
\operatorname{Hom}_{D^{b}(A)}(\mathbf{P}, Y) & \cong \operatorname{Hom}_{A}\left(\mathrm{H}^{0}(\mathbf{P}), Y\right) \\
& \cong \operatorname{Hom}_{A / \mathfrak{A}}\left(\mathrm{H}^{0}(\mathbf{P}), Y\right) \\
& =0 .
\end{aligned}
$$

Thus, $Y \in \mathcal{F}(\mathbf{P})$ as right $A$-module. Note that there is a natural embedding $\operatorname{Ext}_{A / \mathfrak{A}}^{2}(X, Y) \hookrightarrow \operatorname{Ext}_{A}^{2}(X, Y)$ induced by the canonical full embedding $\bmod A / \mathfrak{A} \hookrightarrow$ $\bmod A$. Since $\mathbf{P}$ is splitting, we have $\operatorname{Ext}_{A}^{2}(X, Y)=0$. Thus, $\operatorname{Ext}_{A / \mathfrak{A}}^{2}(X, Y)=0$.

Next, we claim that $\mathrm{H}^{0}(\mathbf{P})$ is a separating tilting $A / \mathfrak{A}$-module.

Indeed, for any pair $X \in \mathcal{F}\left(\mathrm{H}^{0}(\mathbf{P})_{A / \mathfrak{A}}\right), Y \in \mathcal{T}\left(\mathrm{H}^{0}(\mathbf{P})_{A / \mathfrak{A}}\right)$, it is easy to see that $X \in \mathcal{F}(\mathbf{P})$ and $Y \in \mathcal{T}(\mathbf{P})$. Note that there is a monoic map $\operatorname{Ext}_{A / \mathfrak{A}}^{1}(X, Y) \hookrightarrow$ $\operatorname{Ext}_{A}^{1}(X, Y)$. Since $\mathbf{P}$ is separating, $\operatorname{Ext}_{A}^{1}(X, Y)=0$ and so $\operatorname{Ext}_{A / \mathfrak{A}}^{1}(X, Y)=0$. Then, the claim holds.

By Corollary 3.15 and Proposition 5.1, one can get the following consequence.

Corollary 5.2. Let $A$ be a finite dimensional algebra and $\boldsymbol{P}$ be a 2-term splitting and separating silting complex in $K^{b}(\operatorname{proj} A)$. Then rep.dimEnd ${ }_{A}\left(\mathrm{H}^{0}(\mathbf{P})\right)=$ $\operatorname{rep} . \operatorname{dim} A / \mathfrak{A}$.

\section{REFERENCES}

1. M. Auslander, Representation Dimension of Artin Algebras, Math. Notes, Queen Mary College, London, 1971.

2. I. Assem, M. I. Platzeck, S. Trepode, On the representation dimension of tilted and laura algebras, J. Algebra, 296, (2006), 426-439.

3. I. Assem, D. Simson, A. Skowronski, Elements of the Representation Theory of Associative Algebras, vol. 1: Techniques of Representation Theory, London Mathematical Society Student Texts, vol. 65, Cambridge University Press, Cambridge, 2006.

4. H. Abe, Tilting Modules Arising from Two-Term Tilting Complexes, Comm. Algebra, 42(3), (2014), 988-997.

5. A. G. Chaio, S. Trepode, Representation dimension of cluster-concealed algebras, Algebr. Represent. Theor., 16, (2013), 1001-1015.

6. F. U. Coelho, M. I. Platzeck, On the representation dimension of some classes of algebras, J. Algebra, 275, (2004), 615-628.

7. H. X. Chen, W. Hu, tilting modules and representation dimensions, J. Algebra, 323, (2010), 738-748.

8. A. B. Buan, Y. Zhou, A silting theorem, J. Pure Appl. Algebra, 220, (2016), 2748-2770.

9. A. B. Buan, Y. Zhou, Silted algebras, Adv. Math., 303, (2016), 859-887.

10. A. B. Buan, Y. Zhou, Endomorphism algebras of 2-term silting complexes. Algebra Represent. Theory, 21, (2018), 181-194.

11. K. Erdmann, T. Holm, O. Iyama, J. Schröer, Radical embeddings and representation dimension, Adv. Math., 185(1), (2005), 159-177.

12. D. Happel, L. Unger, Representation dimension and tilting, J. Pure Appl. Algebra, 215, (2011), 2315-2321.

13. M. Hoshino, Y. Kato, J. Miyachi, On t-structures and torsion theories induced by compact objects, J. Pure Appl. Algebra, 167(1), (2002), 15-35.

14. K. Igusa and G. Todorov, On the finitistic global dimension conjecture for Artin algebras, in: Representations of Algebras and Related Topics, Fields Inst. Commun.45, Amer. Math. Soc., Providence, RI, (2005), 201-204. 
15. O. Iyama, Finiteness of representation dimension, Proc. Amer. Math. Soc., 131, (2003), 1011-1014.

16. B. Keller, D. Vossieck, Aisles in derived categories, Bull. Soc. Math. Belg., Sér. A, 40(2), (1988), 239-253.

17. F. Oort, Yoneda extensions in abelian categories, Math.Ann. 153, (1964), 227-235.

18. S. Oppermann, Representation dimension of quasi-tilted algebras. J. Lond. Math. Soc., 81(2), (2010), 435-456.

19. R. Rouquier, Representation dimension of exterior algebras, Invent. Math., 165, (2006), 357-367.

20. C. M. Ringel, On the representation dimension of artin algebras. Bull. Inst. Math. Acad. Sin. (N.S.), 7(1), (2012), 33-70.

21. C. C. Xi, Representation dimension and quasi-hereditary algebras, Adv. Math., 168, (2002), 193-212.

22. C. C. Xi, Adjoint functors and representation dimensions, Acta Math. Sin. (Engl. Ser.), 22(2), (2006), 625-640.

College of Applied Sciences, Beijing University of Technology, Beijing, P. R. China, 100124

Current address: Département de mathématiques, Université de Sherbrooke, Sherbrooke, Québec, Canada, J1K 2R1

E-mail address: huyonggang@emails.bjut.edu.cn, huxy2601@usherbrooke.ca 